\title{
A Spin Model for Investigating Chirality
}

\author{
By DaVid A. RABSON AND Stuart A. TRUgman \\ MS B-262, Condensed-Matter Theory Group, T-11 \\ Los Alamos National Laboratory, Los Alamos, NM 87545, USA
}

\begin{abstract}
Spin chirality has generated great interest recently both from possible applications to flux phases and intrinsically, as an example of a several-site magnetic order parameter that can be long-ranged even where simpler order parameters are not. Previous work (motivated by the flux phases) has focused on antiferromagnetic chiral order; we construct a model in which the chirality orders ferromagnetically and investigate the model's behavior as a function of spin. Enlisting the aid of exact diagonalization, spin-waves, perturbation theory, and mean fields, we conclude that the model likely has long-ranged chiral order for spins 1 and greater and no non-trivial chiral order for spin 1/2.
\end{abstract}

May 1995

submitted to Journal of Physics: Condensed Matter

PACS-95 75.10.Jm, 75.30.Ds

Los Alamos Unclassified Release (LAUR) number 95-1734 


\section{Chirality, a Simple Model, and its Classical Ground State}

Among the flood of theories that has issued from the discovery eight years ago of a new class of superconductors, several concern themselves with a competition between antiferromagnetic and chiral order. ${ }^{1}$ These efforts have focused on antiferromagnetic Hamiltonians, often involving frustration. We examine a different way of arriving at chiral order, one using Heisenberg-squared bonds $\left(\mathbf{S}_{i} \cdot \mathbf{S}_{j}\right)^{2}$ (which, for spin greater than $1 / 2$, cannot be reduced to Heisenberg terms). ${ }^{2}$

Given spins $i, j$, and $k$ forming the vertices of a triangle (in figure 1, half a square of a square lattice), we define the (pseudoscalar) chiral order parameter as

$$
\chi_{i j k}=\mathbf{S}_{i} \cdot\left(\mathbf{S}_{j} \times \mathbf{S}_{k}\right)
$$

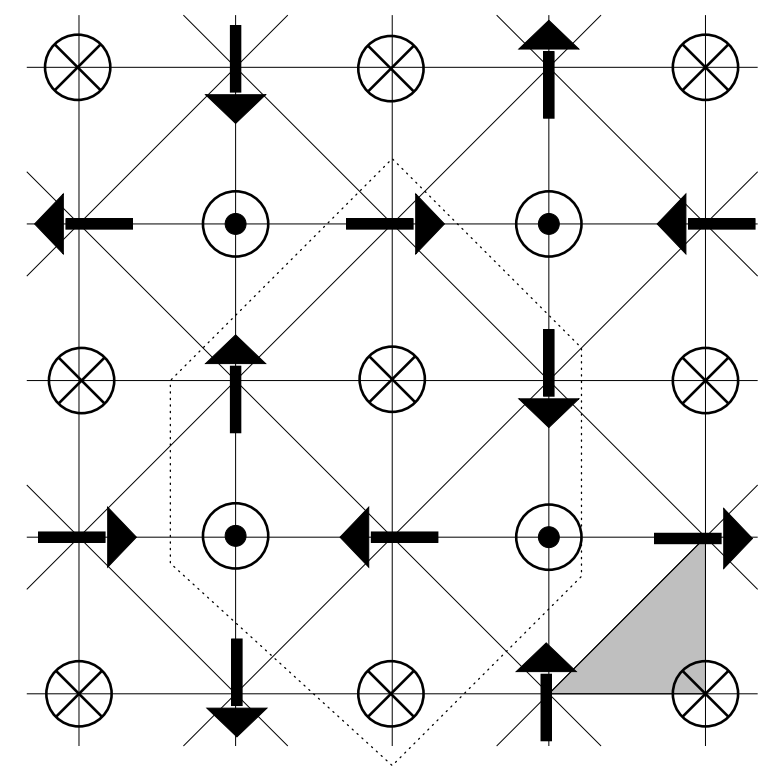

Figure 1. The geometry of the problem: right-, left-, up-, down-, outward- $(\odot)$, and inward-pointing $(\otimes)$ arrows give one classical ground state (others are related by global spin rotation and chirality flip). The lines indicate Heisenberg-squared $\left(\mathbf{S}_{i} \cdot \mathbf{S}_{j}\right)^{2}$ bonds; the antiferromagnetic bonds between opposite-pointing spins are not shown. The sublattice pictured here with spins pointing into or out of the page we call "even" or sublattice 0; sublattices 1, with spins pointing left or right, and 2, up and down, we call "odd." The dotted lines highlight a ground-state unit cell. One example of the plaquettes on which we define chirality is shaded in the lower-right corner.

1 See e.g. Wen, Wilczek, and Zee, 1989; Baskaran, 1989; Gooding, 1990; Marston and Zeng, 1991; Korshunov, 1993; Masui and Betts, 1993; and references contained therein.

2 We note an interest in this "biquadratic" exchange (so called for its second-quantized expression) independent of any resultant chirality: Anderson (1963) (page 168); Harris and Owen (1963); Takhtajan (1982); Affleck et al. (1987); Mitra and Chakraborty (1994). 
We can consider the spin $\mathbf{S}$ either a quantum spin-s or a classical (vector) object; the latter is equivalent to the limit $s \rightarrow \infty$. We will begin with classical spins, constructing interactions in such manner as to ensure a long-range-ordered chiral ground state, then investigate the quantum behavior of the resulting model as we lower the spin from $\infty$ to $1 / 2$. Understanding the ground state as a function of spin may later prove useful in more realistic models. ${ }^{3}$

For convenience, we use a square lattice. There are two translationally-related ways of drawing diagonals across squares to split the lattice into triangular plaquettes while preserving the point group of the whole lattice. We will construct a Hamiltonian such that $\chi$ on the triangles outlined in figure 1 takes its maximal absolute value, while on the triangles formed by drawing diagonals the other way it has zero value. We note that this will result in chiral-ferromagnetic order, in that $\chi$ has the same sign everywhere on the lattice, as opposed to the chiral-antiferromagnetism considered in the references listed in footnote 1. (Antiferromagnetic chirality is motivated in part by an analogy to the flux phase of Marston and Affleck, 1989, in which a gauge field living on the bonds of a square lattice induces a staggered magnetic-like field on the square plaquettes. A ferromagnetic chirality, in contrast, would correspond to a gauge field that cancels everywhere in the interior, leaving a net circulation around the outside of a sample.)

Viewed as classical vectors, the spins on the vertices of a triangle maximize the absolute value of $\chi$ for that triangular plaquette when they are mutually perpendicular: we therefore begin with a Heisenberg-squared term $\left(\mathbf{S}_{i} \cdot \mathbf{S}_{j}\right)^{2}$ for all neighboring sites $i, j$ and those pairs of next-neighbor sites joined by diagonals in figure 1 . By itself, however, this would yield local chiral plaquettes with no correlation in sign between one plaquette and its neighbors. To enforce such correlation, it suffices to add antiferromagnetic bonds linking those nearest spins that we wish (see figure 1) to align antiferromagnetically: specifically, we add next-neighbor antiferromagnetic bonds across the square diagonals that do not already have Heisenbergsquared bonds and between "odd" third-neighbor pairs, where we define as odd those sites in figure 1 from which emanate eight rays against the four at each even site. To maintain

\footnotetext{
3 As an example, $\mathrm{URu}_{2} \mathrm{Si}_{2}$ has a large specific-heat jump at a presumably magnetic transition, yet almost no antiferromagnetic ordering, a discrepancy Gor'kov and Sokol (1992) and Agterberg and Walker (1994) explain with chiral order parameters.
} 
the two kinds of terms, those quartic in spin and those quadratic, equal in strength for all values of spin, we divide each term by spin to the appropriate power:

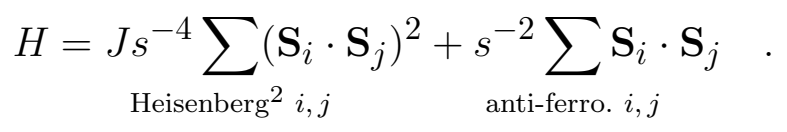

With this Hamiltonian, one easily confirms the classical ground state of figure 1, up to a global spin rotation and the global sign of chirality (plus or minus).

The parameter $J$ controls the relative importance of the quartic and quadratic terms. For classical spins, the ground state is the same for any positive value. With the Heisenbergsquared bonds turned off, $J \rightarrow 0$, the problem splits into three entirely independent antiferromagnetic square sublattices.

The chirality, (1), is a pseudoscalar, and the Hamiltonian locally encourages it to take its full value, either positive or negative. In this sense, it more closely resembles an Ising than a vector spin: even as long-wavelength twisting modes might cause spin-spin correlations within each antiferromagnetic sublattice to decay exponentially with distance, the individual sublattices could remain locally mutually perpendicular, preserving the sign and strength of the chirality to a higher temperature. We therefore expect that chiral order will be more robust than spin order unless quantum-mechanical peculiarities at small spin favor another phase.

At zero temperature, the antiferromagnetic bonds alone $(J=0)$ suffice to order the ground state in a trivial way: with the three decoupled sublattices (left-right, up-down, inout in figure 1) free to assume any relative orientation, long-range antiferromagnetic order on each will correlate $\chi$ at one plaquette with $\chi$ on any plaquette arbitrarily far away. As we shall demonstrate, however, thermal fluctuations destroy this freedom ("order from disorder" 4 ) and with it, for spin-1/2, the chirality. For spins 1 and higher, the same fluctuations instead favor chiral order. In the zero-temperature diagonalizations to follow, we shall search for evidence of ordering 1 . in excess of the trivial order and 2. for $J \gtrsim 1$, away from where we expect the trivial mechanism to operate.

\section{Exact Diagonalization of Small Clusters}

The classical $(s=\infty)$ ground state is fixed, by our construction. We examine in three

\footnotetext{
4 Villain, 1979, Villain et al. 1980, Henley 1989.
} 
ways the question of what happens as $s$ is lowered to more physical values: first by exactly diagonalizing the problem for small numbers of sites, then in a spin-wave (" $1 / s ")$ calculation, and finally by considering related problems motivated by lingering doubts on the fate of spin one-half.

We impose periodic boundary conditions. While there are only two inequivalent sites in the Hamiltonian, the classical ground state (see figure 1) has a unit cell of eight; we would expect (and indeed verify) that any classically frustrated cluster (for instance, one with other than a multiple of eight sites) would have depressed chiral order. Because the size of the Hilbert space grows exponentially with the number of sites and very fast with the spin as well, we cannot examine with exact diagonalization clusters as large we might wish. Without loss of generality, we limit our diagonalization to the space with zero total spin projected along the $\hat{\mathbf{z}}$ axis $\left(S_{\text {total }}^{z}=\sum_{\text {sites } i} S_{i}^{z}=0\right)$. We furthermore assume the nondegeneracy of the ground state, so that we may limit our attention to momenta 0 and $\pi$ along the two spatial directions. ${ }^{5}$ While in principle we could further reduce the size of the space by restricting ourselves to the total spin-zero sector $\left(\mathbf{S}_{\text {total }}=\mathbf{0}\right)$, employing the valencebond (singlet) basis (Ramasesha and Soos, 1984; Chang et al., 1989), its non-orthogonality makes it impractical for calculating eigenvectors or correlation functions. ${ }^{6}$ Once a basis with the proper symmetry has been constructed (following the usual procedure of summing with proper phases the states in the symmetry element's orbit), it is straightforward to find the

5 This assumption holds for clusters small enough that we could vary momentum.

6 The aforementioned valence-bond basis works well for some one-dimensional problems with local (nearest or at worst second- or third-neighbor) interactions. However, to evaluate two-dimensional interactions requires either an expansion of the operators (see Ramasesha and Soos) or the application of a diagram-untangling rule. The latter is more efficient but still results in the generation of a large number of diagrams that must be contracted. More significantly, the non-orthogonality of the valence-bond basis converts the eigenproblem into the diagonalization of a non-Hermitian matrix, precluding the use of the Lanczos algorithm. (Existing non-symmetric Lanczos algorithms do not work well, while orthogonalizing the basis would prove as expensive as diagonalizing the Hamiltonian, as well as destroying sparseness.) Once the ground-state wavefunction has been found, nonorthogonality makes the evaluation of observable operators an order- $n^{2}$ operation ( $n$ the basis size), destroying the advantage of shrinking the Hilbert space. The difficulties become worse with larger spin: the generalized basis described by Chang et al. (1989), while appropriate for calculating energies, is unsuitable for the evaluation of expectation values and must first be expanded. The number of expansion steps grows exponentially with the number of sites and so is not practical. 


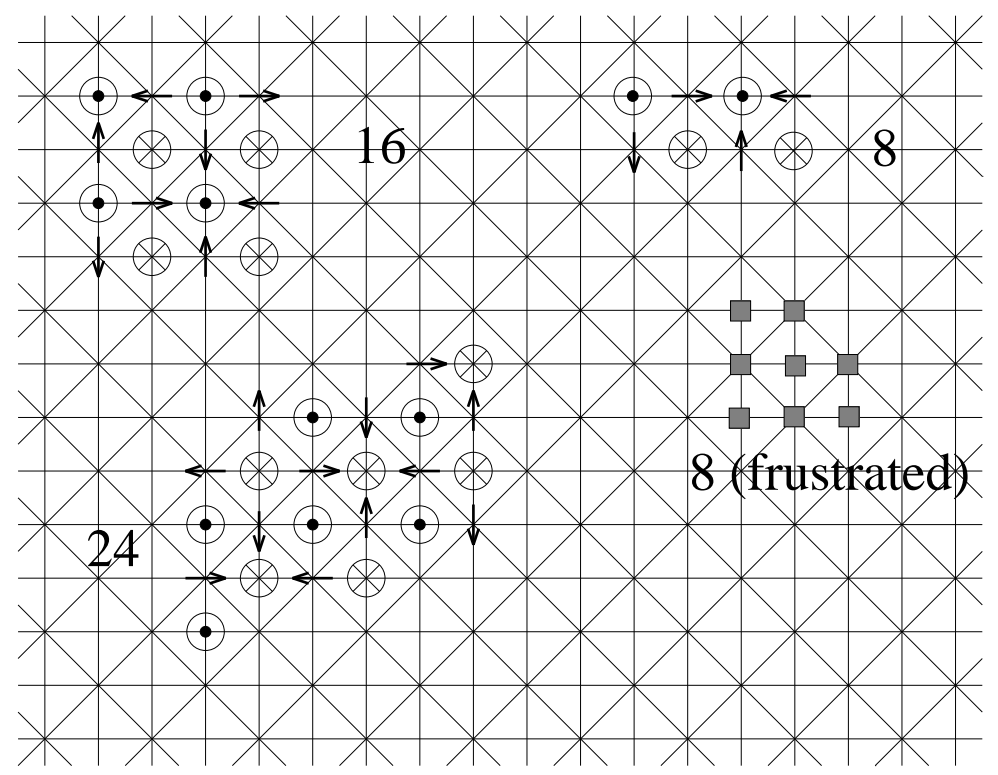

Figure 2. Exact diagonalization: we use clusters of 8, 16, and 24 spins, for which we show classical ground states. For purposes of comparison, we also use an additional eight-site cluster frustrated by periodic boundary conditions.

ground state with the Lanczos method.

For unfrustrated 8-, 16-, and 24-site spin- $1 / 2$ clusters (figure 2 ), and for a range of spins and ratios $J$ of the two bond strengths, we calculated the chirality-chirality correlations at the largest separations possible, with the results shown in figure 3 . The spin- $1 / 2$ clusters (two bottom traces) show their largest correlation, still less than $15 \%$ of the maximum attainable by three spins one-half, for negative $J$. This value lies barely above the $J=0$ correlation of 0.1361 (16 spins) of entirely uncoupled spin- $1 / 2$ sublattices. Since the actual peak ordering occurs for $J<0$ and does not rise substantially above the "trivial" ordering value, we conclude that spin- $-1 / 2$ has no non-trivial chiral ordering. ${ }^{7}$

As $J$ increases, the correlations fall, dropping abruptly to zero at .93 (for 16 spins, or .89 for 24$)$, coincident with a partial ferromagnetic transition. While for the non-zero part of the curve the ground state forms a spin singlet, in an intermediate regime $(J=$ about .93-1 for 16 spins, .89-1 for 24), the cluster carries spin one less than ferromagnetic, spin 7

7 For uncorrelated but ordered classical sublattices, averaging over relative angles gives $\sqrt{\chi \chi}=\sqrt{2 / 9} \approx 47 \%$. Quantum fluctuations reduce this to .1113 (normalized) in spinwave theory for spins $1 / 2$, close to the .1361 quoted above and calculated for the actual antiferromagnetic correlations measured in the cluster. We shall later provide evidence that in the small- $J$ regime that has the "trivial" order, thermal fluctuations will destroy it. 


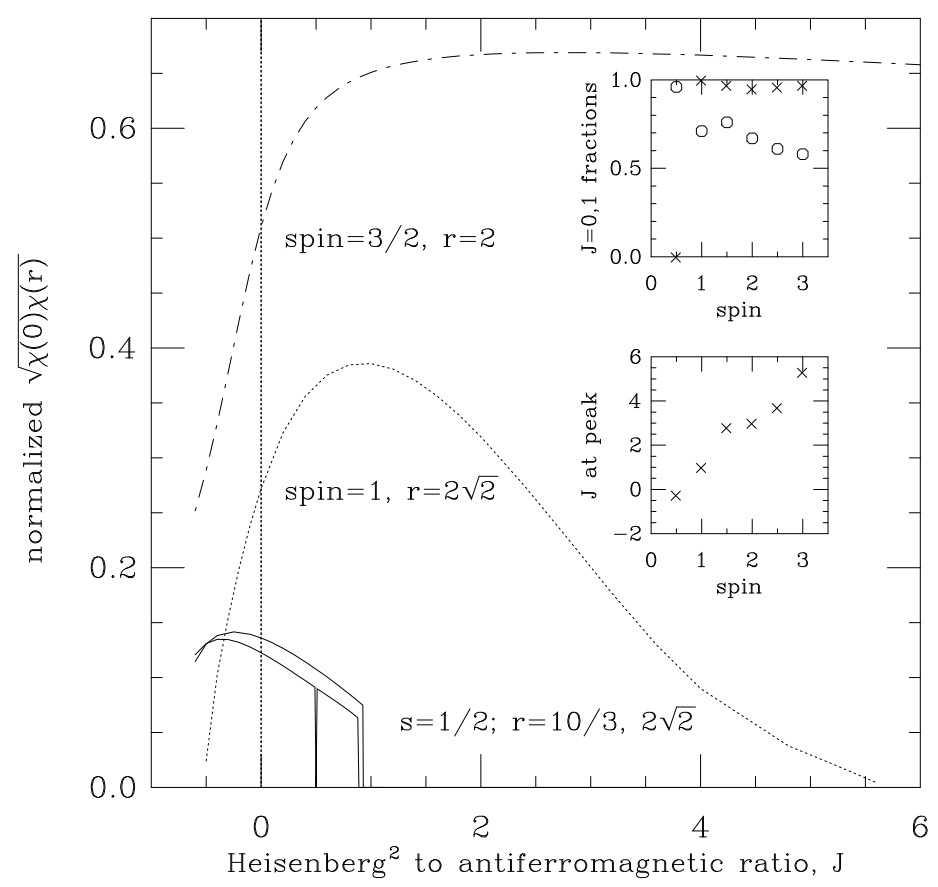

Figure 3. Exact diagonalizations varying the ratio $J$ of the two bond strengths for spins $1 / 2$, 1 , and $3 / 2$ : we plot the square root of normalized chirality-chirality correlation as a function of $J$. Unity on the vertical axis represents the maximum eigenvalue of $\chi(123)$. The two lowest plots (solid lines), showing less than $15 \%$ maximum chirality, are for spin $1 / 2$. Note that the maximum occurs below $J=0$ : in other words, without the benefit of Heisenberg-squared coupling. This level of chirality is expected for uncorrelated antiferromagnetic lattices and should be subtracted (see footnote 7). The lower of the two solid traces is for 24 spins (it dips once at $J=.5$, where a ferromagnetic state is exactly degenerate) and a distance of $10 / 3$, the largest possible in the cluster. The upper spin- $1 / 2$ trace is for 16 sites and a distance of $2 \sqrt{2}$. These curves drop suddenly to zero correlation at $J=.89$ (24 sites) or .93 (16 sites), corresponding to a partial ferromagnetic transition. The ground state becomes fully ferromagnetic around 1.0. The next trace up (dotted line) is for 16 spin- 1 sites; it peaks around $J=1$, where it shows nearly half again as much chiral order as when zero $J$ decouples the sublattices. Note that the order falls continuously to zero as $J$ approaches about 6 ; there is no ferromagnetic transition for $J$ as large as 100 . We take this curve as evidence of at least short-range order, possibly long-ranged. The top curve (dashed) shows the chirality correlation at a distance of 2 for spin- $3 / 2$ on an eight-site cluster.

The $J$ corresponding to the peak correlation shifts higher with larger spin, as indicated in the lower inset for spins up to 3. Countering this trend, however, the curves become increasingly flat. As shown by the crosses $(x)$ in the upper inset, for spins greater than $1 / 2$, the correlation at $J=1$ appears to saturate near to the maximum for each spin. Conversely, the fraction of peak correlation realized with the Heisenberg-squared coupling turned off $(J=0, \bigcirc)$ starts close to unity for spin- $1 / 2$ but quickly drops to a level compatible with random orientations. Since the classical problem $(\operatorname{spin} \rightarrow \infty)$ would have full correlation for all $J>0$ but only $47 \%$ correlation for $J=0$ (see footnote 7 ), we take this inset as evidence for a possible order-disorder transition between spin- 1 and spin-1/2.

for 16 sites or 11 for 24 . Above $J=1$, both clusters become fully ferromagnetic. It is not surprising that the Hamiltonian should behave differently for spin $1 / 2$ than classically, for 
the Heisenberg-squared term $\left(\mathbf{S}_{i} \cdot \mathbf{S}_{j}\right)^{2}$ reduces in the former case to a simple ferromagnetic interaction, $\frac{3}{16}-\frac{1}{2} \mathbf{S}_{i} \cdot \mathbf{S}_{j}$. Even at the value of $J$ for which the correlation reaches a maximum, extrapolation of the correlation as a function of distance (figure 4) is compatible with no more than the trivial chirality correlations expected for essentially decoupled lattices.
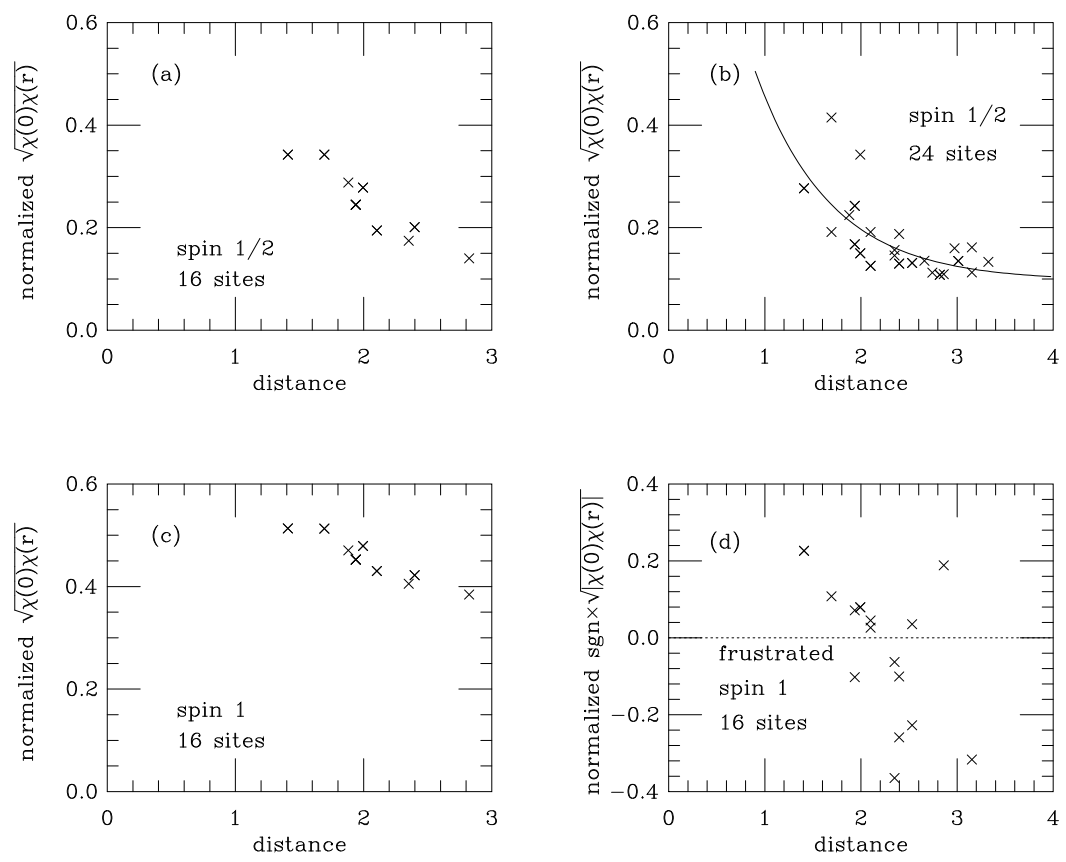

Figure 4. Distance dependence of chirality correlations for spins $1 / 2$ (upper row) and 1 (lower). The vertical axes are normalized as in figure 3. Least-squares fits to $c_{0}+$ $\gamma \exp (-x / \xi), x$ the distance, were inconclusive for the 16 -site clusters. (The root-meansquare variances were larger than the fitting parameters.) However, from (b) we see 24 already approaching an asymptotic correlation $c_{0}=0.10 \pm .04$, compatible with the predicted 0.1113 (footnote 7 ), and rapidly, with a coherence length $\xi=.8 \pm .4$ equal to or smaller than a lattice spacing. Each case (a)-(c) fixes $J$ at its peak value from figure 3. In (d) we plot the correlation for a frustrated spin-1 cluster. Note the smaller absolute correlations as well as the greater scatter.

For spin 1, we found in the 16-site cluster no ferromagnetic transition for any value of $J$ up through 100. Instead of dropping suddenly, the chirality-chirality correlation in figure 3 falls continuously to zero as $J$ approaches about 6 . While we cannot, as for spin $1 / 2$, write the quartic term as purely ferromagnetic, it can be written simply as the sum of a constant, a ferromagnetic piece, and a site-interchange operator, $\left(1-\mathbf{S}_{i} \cdot \mathbf{S}_{j}+\mathrm{X}_{i j}\right){ }^{8}$ We interpret the

8 Alternatively, one can write the quartic interaction between quantum spin-1 coherent states (ground-state eigenvectors of operators $-\mathbf{S} \cdot \hat{\mathbf{n}}(\theta, \phi)$ for some $\hat{\mathbf{n}}$ on the unit sphere) as the sum of again a ferromagnetic piece, a constant, and a Heisenberg-squared term favoring 


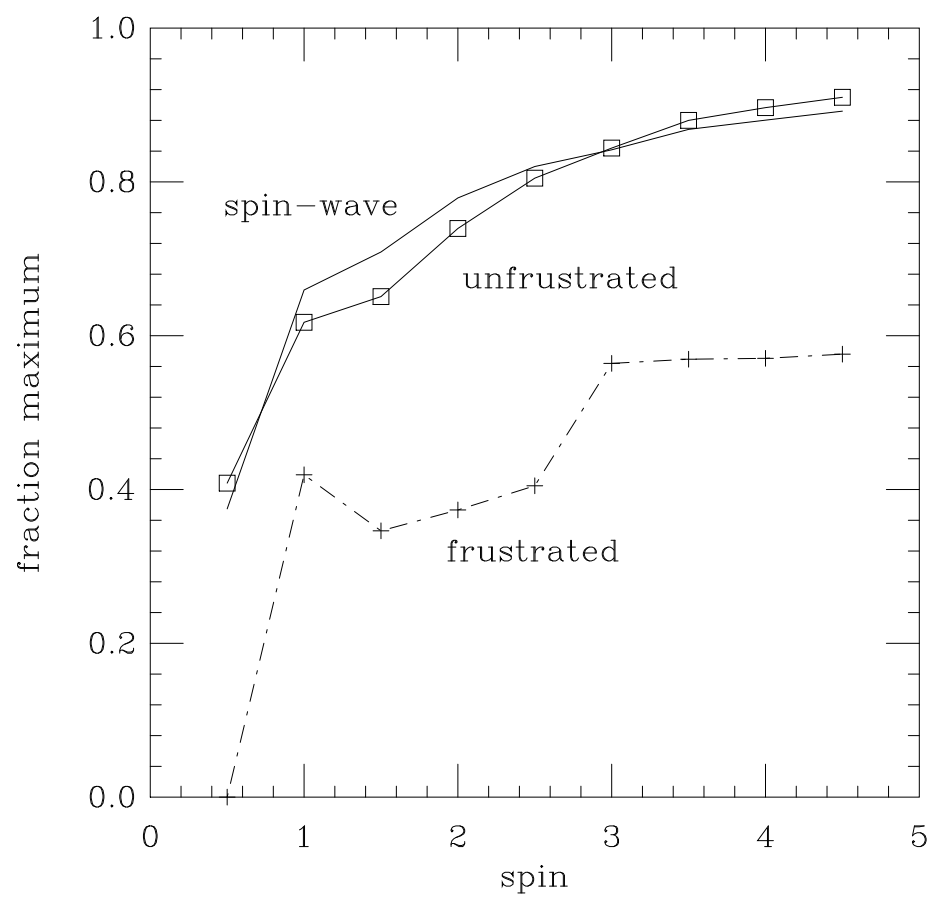

Figure 5. Exact diagonalization and spin-wave theory for $J=1$. The middle trace, marked with squares and labeled "unfrustrated," plots $\sqrt{\chi(0) \chi(2)}$, normalized to its maximum possible value, on the eight-site cluster as a function of spin ( 2 is as far away as two plaquettes get on this cluster; results for $\sqrt{2}$ do not differ markedly). Above it (solid line), we plot $\chi$ from spin-wave theory. The close agreement of the two bolsters our confidence in the results to some extent. Below these two lines, we plot $\sqrt{\chi(0) \chi(\sqrt{2})}$ on the frustrated eight-site cluster.

falling order as a ferromagnetic tendency that still carries some weight at spin 1 but vanishes in the limit of infinite spin.

Figure 5 displays the spin dependence of the largest-distance chirality-correlation function achievable on the unfrustrated eight-site geometry for spins between $1 / 2$ and $9 / 2$; all correlations are shown normalized to the largest quantum-mechanically possible value for $\chi$ on three sites, and $J$ is fixed at 1 . For comparison, we also show the results of the spinwave calculation for the correlation at infinite separation, while contrasting the normalized chirality-chirality correlation for a frustrated geometry in which even the classical system cannot have full chiral order on every plaquette.

These results cannot be considered conclusive, given the small cluster sizes and the inability (with so few sizes from which to choose) to apply finite-size scaling. However, they chirality: $\left\langle\left(\mathbf{S}_{0} \cdot \mathbf{S}_{1}\right)^{2}\right\rangle=-(1 / 2) \cos \psi+5 / 4+(1 / 4) \cos ^{2} \psi$, with $\psi$ the relative angle between the two coherent states. 
at least support the suspicion that the system might have non-trivial long-range order ${ }^{9}$ for spins greater than $1 / 2$.

\section{Spin-wave calculation}

Spin-wave theory (for a review, see Manousakis, 1991) assumes a ground state close to the classical state, with dynamical variables, supposed small, indicating deviations. The theory constitutes an expansion in these small, dimensionless variables divided by the spin, which we wish to vary. As such, it cannot say anything about the properties of a system in a regime where the assumed ground state fails to approximate the true state, but it does give a rough idea of where the failure occurs, in our case the approximate spin value below which the classical chiral ground state is no longer an appropriate starting point.

We diagonalize not in the traditional Holstein-Primakov Bose operators but in a more convenient linear combination of them. Our methods, while not differing in spirit from usual treatments, may be of use to others, so we outline them in the appendix.

As figure 5 shows, the spin-wave results agree fairly closely with the finite-size numerical correlations. The two calculations measure somewhat different objects. The diagonalization looked at $\langle\chi(0) \chi(r)\rangle$ for as large an $r$ as could fit in a cluster. It could not directly measure the "chiral polarization" $\langle\chi(0)\rangle$, since there is nothing to break the symmetry of positive and negative chirality. The spin-wave calculation, in contrast, breaks that symmetry from the outset and asks how large the deviation is: it does measure ${ }^{10}\langle\chi(0)\rangle$. A deviation from order calculated by the spin waves greater than the maximum value of the order itself would indicate the breakdown of spin-wave theory and probably the loss of long-range order. The figure shows the chirality for $J=1$ in all cases greater than zero, although down at spin $1 / 2$ the deviation from full chirality is more than half.

The actual value $s$ of spin enters into spin-wave theory only in determining when the theory breaks down (viz., when the deviations grow larger than the maximum $\chi$ ); the system

\footnotetext{
9 That is, larger than the zero-temperature ordering of uncorrelated sublattices, in a regime of $J$ 's for which the trivial mechanism should fail to operate.

10 The chiral-chiral correlation in the spin-wave theory for this problem, $\langle\chi(0) \chi(r)\rangle$, is essentially the square of this, approximately independent of $r$. In other words, the deviationdeviation correlation is negligible. This is confirmed by looking at the deviation $\Delta \chi(\mathbf{k})$ as a function of wavenumber $\mathbf{k}$ : it is nearly flat, giving a deviation correlation sharply peaked at $\mathbf{0}$ in real space.
} 
can't know about the quantum-mechanical ferromagnetic tendency of $\left(\mathbf{S}_{i} \cdot \mathbf{S}_{j}\right)^{2}$ at small spins. Thus we would expect the spin-wave calculation to underestimate the deviations for $s=1 / 2$.

\section{Is the order long-ranged?}

The spin-wave theory of figure 5 fails to find any value of spin for which fluctuations completely destroy chiral order, although at spin 1/2 most of the order has been eroded. Considering the uncertainties (see the appendix) of extending spin-wave theory to spin $1 / 2$, and the lack of order at $J \gtrsim 1$ in the diagonalizations, we may well believe that the spin-1/2 system is not ordered in this regime.

For spin 1, it is easier to come to a conclusion. While figures $4 \mathrm{c}$ and $4 \mathrm{a}$ suggest a greater extrapolated order for spin 1 than for spin 1/2, as well as a longer decay length, the statistics do not permit a clear interpretation. However, the unambiguously ordered prediction of spinwave theory, combined with reasonably close quantitative agreement of the two calculations, suggests that spin 1 is ordered. Since the peak ordering occurs at $J=1$ we can be confident the effect is not caused by fluctuations of uncoupled lattices, as with spin $1 / 2$. (We shall estimate that the lattices should couple for $J \gtrsim 0.5$ or even for smaller $J$.) We believe that higher spins are ordered as well.

The Ising-like character of the chiral order parameter further bolsters the conclusion. Except in the identified ferromagnetic state found for large enough $J$ when spin is $1 / 2$, we see in the exact diagonalization antiferromagnetic order on the antiferromagnetically-coupled sublattices. Since we expect Ising-like order to be at least as robust as spin order, we would have to be surprised by the absence of the former in the presence of the latter. Only when spin is $1 / 2$ do we find a transition to a competing magnetic state, which then destroys chirality.

\section{No non-trivial long-range order for spin-1/2}

We consider another approach to the question of spin $1 / 2$ in the troublesome regime of smaller $J$, where lattice decoupling appeared to induce the "trivial" ordering of footnote 7. (For larger $J$, a ferromagnetic transition killed even that kind of ordering.) We will present evidence that any infinitesimal temperature should destroy chiral ordering for spin $1 / 2$. This is a stronger statement than the analogous assertion for magnetic ordering in the Heisenberg model for two reasons: chirality has an Ising-like rather than a continuous 
symmetry, so the Mermin-Wagner theorem does not apply; furthermore, we shall find that infinitesimal temperature kills even the local chirality on individual plaquettes, not just chirality correlation.

Restricted to spins $1 / 2$, the Hamiltonian becomes (up to a constant)

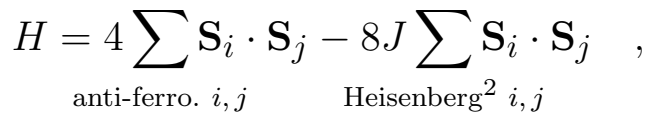

the classically frustrated behavior of which we may scrutinize for hints about the quantum system. One cannot always hope to guess the correct ground state for a frustrated classical system, so we used a straightforward numerical minimization procedure on finite clusters of classical spins with periodic boundary conditions (open boundaries produced spurious chiral phases).

Equation (3) follows from (2) by an operator identity, not by an approximation. The approximation consists in treating the spins in (3) classically. ${ }^{11}$ Letting the classical spins have unit length (instead of length $1 / 2$ ), we divide the coefficients in (3) by four.

Numerically minimizing the energy of the configuration with respect to the angles of the classical spins, we find three regimes of $J$. Without ferromagnetic bonds $(J=0)$, the system consists of three antiferromagnetic lattices with undetermined relative angles, a degeneracy that persists up through a $J$ of approximately 0.5 , when states with mixed chiral order (possibly long ranged) take over: these states differ from the ferromagnetic chirality we saw at higher spins in alternating positive, negative, and zero values on adjacent plaquettes. Similarly, the ferromagnetic state that prevails at infinite $J$ (turning off the antiferromagnetic bonds) appears to be stable against canting deformations (at zero temperature) down to a $J$ of around 0.97 , in remarkable agreement with the diagonalizations of figure 3 .

These classical results suggest no chiral order of the sort we seek, but we can go a little further by examining the effects of quantum fluctuations on the undetermined ground state we found for small $J$. Before addressing quantum fluctuations directly, however, we will look at their classical relatives, thermal fluctuations.

11 This treatment is identical to a variational calculation in the subspace of spin- $1 / 2$ coherent-state products (see footnote 8). 


\section{low-temperature expansion}

In the regime $0<J \lesssim 0.5$, where the classical model found a degenerate ground state, we wish to examine whether fluctuations will favor one relative sublattice orientation over the others. The classical theory of multiple phase transitions begins with a low-temperature expansion (Domb, 1960, Fisher and Selke, 1981), which will serve as an inspiration for our rather simpler purposes. Non-zero temperature will induce low-energy excitations, which we may view either as long-wavelength twists in the antiferromagnetic lattices or as very small deviations, tips, of isolated spins (in an Ising model, these would be "flips" costing a discrete energy; see Fisher and Selke, 1981). Because of the analogy to the Ising model, we present the calculation from the second viewpoint, although we have arrived at the same result the first way, too.

A single isolated tipped spin will not prefer any relative lattice orientation, since each ferromagnetic bond linking the affected spin with a spin in another sublattice is canceled by an equal bond to a third spin, pointing exactly oppositely, on that other sublattice. When two neighboring spins on different sublattices tip simultaneously, however, we shall see that they favor a particular orientation of their two sublattices.

Consider tips of a single spin on the even sublattice (0) (refer to figure 1) and of a neighboring spin on either of the others, say 1. (One obviously gets the same results for sublattices 0 and 2 and in fact also for sublattices 1 and 2.) We fix the untipped spins in sublattice 0 to point in the positive and negative $\hat{\mathbf{z}}$ directions, while sublattice 1 points some angle $\theta_{1}$ from the $\hat{\mathbf{z}}$ axis toward the $\pm \hat{\mathbf{x}}$ direction. See figure 6 . If the tipping angles away from their respective preferred axes are both some small number $\delta$, while the azimuthal

angles relative to the sublattice directions are $\phi^{(0)}$ and $\phi^{(1)}$, we can add up all the broken bonds to get a cost for the excitation of

$$
\Delta E=4 \delta^{2}+2 J \delta^{2}\left(\cos \theta_{1} \cos \phi^{(0)} \cos \phi^{(1)}+\sin \phi^{(0)} \sin \phi^{(1)}\right)
$$

where we have kept only the leading power of the small tipping angle $\delta$.

The first term represents the cost of one spin tip on each of the two otherwise antiferromagnetic lattices; it will serve as an expansion parameter if we can fix $\delta$ at some small value. (Without this approximation, connected tips of arbitrarily many spins by arbitrarily small $\delta$ 's would carry the same weights, something we cannot handle. There is some subtlety in 


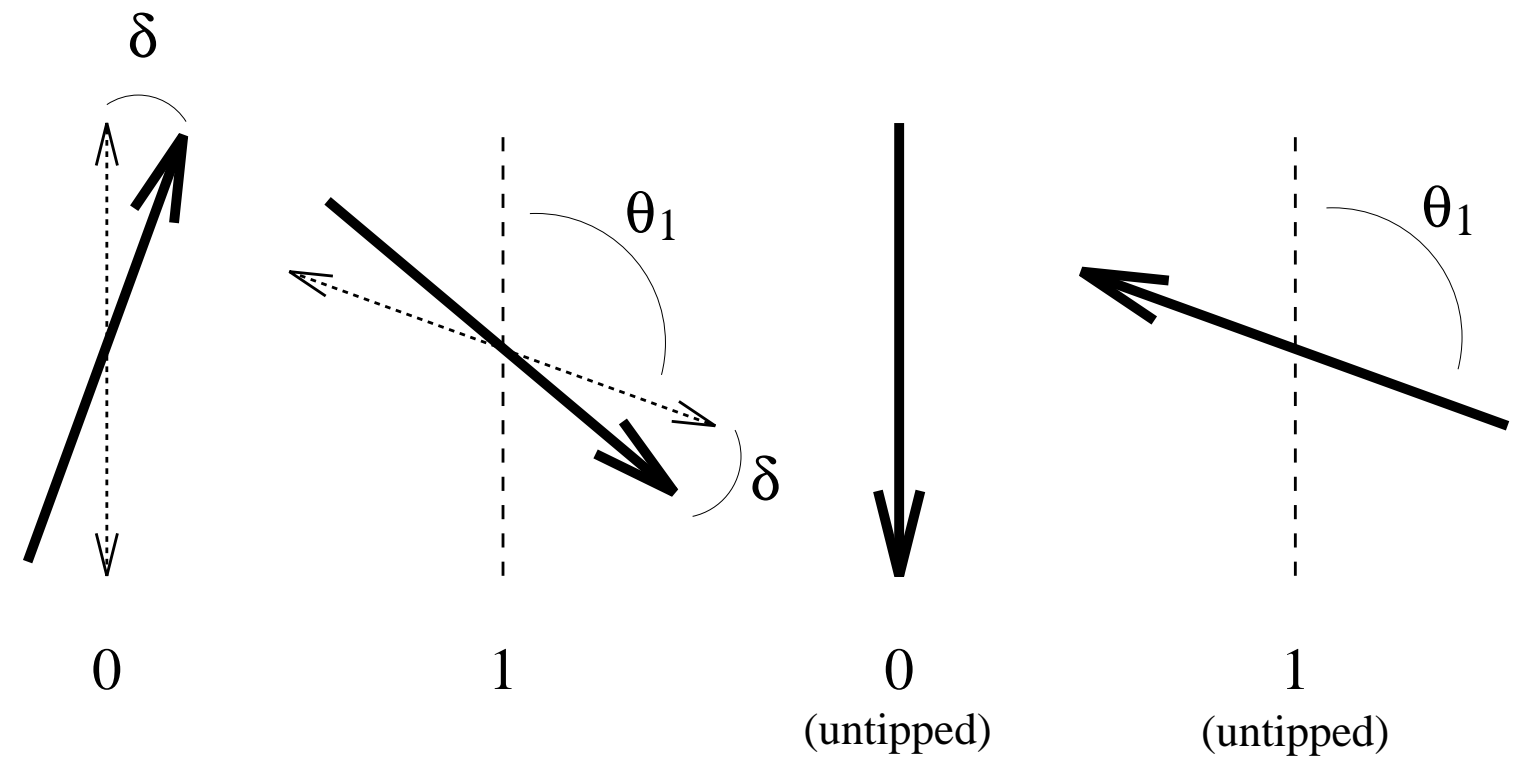

Figure 6. Low-temperature expansion: we consider two of the three sublattices. Spins on sublattice 0 point straight up or down (one is shown as "untipped"), while those on sublattice 1 point an angle $\theta_{1}$ away from the vertical. Thermal fluctuations sometimes cause two neighboring spins to tip an angle $\delta$ away from their equilibrium orientations, indicated for the two spins on the left by double-arrowed thin lines. Not pictured are the azimuthal angles $\phi^{(0)}$ and $\phi^{(1)}$ about these thin lines.

the order in which temperature, $\delta$, and the quantization of azimuthal angles go to zero as well as in the linked-cluster theorem; see Domb, 1960.)

Given an excitation cost, we add Boltzmann weights from all values of the angles $\phi^{(0)}$ and $\phi^{(1)}$ to get a change in the free energy, due to the possibility of such excitations, proportional to

$$
\begin{aligned}
f\left(\theta_{1}\right) & =-k_{\mathrm{B}} T \int_{-\pi}^{\pi} d \phi^{(0)} \int_{-\pi}^{\pi} d \phi^{(1)} \exp \left(-\beta \Delta E\left(\phi^{(0)}, \phi^{(1)}\right)\right) \\
& =-4 \pi^{2} k_{\mathrm{B}} T e^{-4 \beta \delta^{2}} I_{0}(\alpha) I_{0}\left(\frac{b}{4 \alpha}\right)
\end{aligned}
$$

where $\alpha=\sqrt{\left(a+b / 2+\gamma \cos \theta_{1}\right) / 2}, a=\gamma \cos ^{2} \theta_{1}, b=\gamma \sin ^{2} \theta_{1}, \gamma=4 \beta^{2} J^{2} \delta^{4}$, and $I_{0}$ is a Bessel function of imaginary argument. One verifies numerically or graphically that $f\left(\theta_{1}\right)$ has its minima at 0 and $\pi$, meaning that sublattices 0 and 1 prefer, in any infinitesimal temperature, to be aligned. ${ }^{12}$ Since the same is true of 0 and 2 and of 1 and 2, every spin in the problem will point very nearly in plus or minus the same direction, and there will be no chirality, not even locally.

12 One may numerically integrate out the parameter $\delta$ without changing this conclusion. 


\section{treating quantum fluctuations as perturbations}

We now replace thermal fluctuations with those due to quantum mechanics, for which purpose we expand effectively in lattice fluctuations. For antiferromagnetic fluctuations, one typically writes $\mathbf{S}_{i} \cdot \mathbf{S}_{j}=S_{i}^{z} S_{j}^{z}+\frac{1}{2} \lambda\left(S_{i}^{+} S_{j}^{-}+S_{i}^{-} S_{j}^{+}\right)$, where quantum mechanics requires that $\lambda$ should equal unity, while $\lambda=0$ is essentially classical.

We shall later see that fluctuations away from the Néel state, present in the antiferromagnetic sublattices, will order the randomly-oriented lattices. First, however, we consider fluctuations in the ferromagnetic (spin-1/2-Heisenberg-squared) bonds.

Isolated fluctuating spins in a given sublattice cannot break the degeneracy, but as in the thermal case, connected fluctuations of neighboring spins on different sublattices can. Consider sublattice 0 to be quantized in the $\hat{\mathbf{z}}$ direction, while the spins in sublattice 1 are quantized along an axis an angle $\theta_{1}$ off $\hat{\mathbf{z}}$. The spins in this second sublattice are in pure states only if we rotate by $\theta_{1}$ the coordinates we use to describe them: thus for site $i$ in sublattice 0 and site $j$ in sublattice 1 ,

$$
\begin{aligned}
& \mathbf{S}_{0 i} \cdot \mathbf{S}_{1 j}= \\
& \quad\left\{\cos \theta_{1} S_{0 i}^{z} S_{1 j}^{\prime z}\right\} \\
& \quad+\frac{\lambda}{2}\left\{-\sin \theta_{1} S_{0 i}^{z}\left[S_{1 j}^{\prime+}+S_{1 j}^{\prime-}\right]+\sin \theta_{1}\left[S_{0 i}^{+}+S_{0 i}^{-}\right] S_{1 j}^{\prime z}\right\} \\
& \quad+\frac{\lambda}{4}\left\{\left(\cos \theta_{1}-1\right)\left[S_{0 i}^{+} S_{1 j}^{\prime+}+S_{0 i}^{-} S_{1 j}^{\prime-}\right]+\left(\cos \theta_{1}+1\right)\left[S_{0 i}^{+} S_{1 j}^{\prime}+S_{0 i}^{-} S_{1 j}^{\prime+}\right]\right\}
\end{aligned}
$$

where the primes indicate rotated operators. The first term is just the classical Ising piece. We have multiplied the remaining "quantum-mechanical" terms by $\lambda .{ }^{13}$ Each of the second set of terms flips a single spin, thereby contributing to the energy shift in second-order Rayleigh-Schrödinger perturbation theory. However, each spin acted on by one of the raising or lowering operators in these terms has one unperturbed neighbor (acted on by $S_{0 i}^{z}$ or $\left.S_{1 j}^{\prime z}\right)$ pointing up in the appropriate lattice and one neighbor pointing down (here we ignore antiferromagnetic fluctuations). These sum to zero; therefore none of the terms in the second set contributes.

13 Setting $\lambda=1$ eliminates the anisotropy essential to this calculation, but as the parameter is tuned closer to its true quantum mechanical value, one would also have to turn on the antiferromagnetic fluctuations. 
Each of the terms in the third set of braces contributes (with the same energy denominator) in second-order perturbation theory; the energy shift is proportional to

$$
-\left(\cos \theta_{1}-1\right)^{2}-\left(\cos \theta_{1}+1\right)^{2} \propto-\cos ^{2} \theta_{1}-\text { const }
$$

which lowers the energy most when $\theta_{1}=0, \pi$. Quantum fluctuations, therefore, like the classical thermal ones, predict alignment of the sublattices and hence a lack of chiral ordering in the regime considered.

\section{A mean-field theory agrees, too}

In a similar spirit, consider the four spins shown in figure 7: spins 1 and 2 sit on one sublattice and are joined by an antiferromagnetic bond, while 3 and 4 sit on another sublattice and are similarly joined. Ferromagnetic bonds link spins across sublattices, while a mean field $\pm 12 h \hat{\mathbf{z}}$ keeps 1 and 2 pointing in the $\pm \hat{\mathbf{z}}$ directions, while a rotated version of the same keeps 3 and 4 pointing in the $\pm\left(\cos \theta_{1} \hat{\mathbf{z}}+\sin \theta_{1} \hat{\mathbf{x}}\right)$ directions. (The 12 comes from three antiferromagnetic bonds per site not included in figure 7 but contributing through the mean field). We assume the mean ferromagnetic field is small enough to be ignored, both because $J$ is small and because the contributions from neighbors will nearly cancel. Treated classically, this cluster shows no preference for any relative orientation of the sublattices. Quantum-mechanical fluctuations, however, once again break the degeneracy.

We (or rather our computers) easily diagonalize the four-spin Hamiltonian with the extra mean-field terms; we then set $h$ equal to the staggered magnetization and iterate until $h$ converges. For $J$ smaller than about 1.6 (quite a large value), $h$ converges to a non-zero value not too far from full antiferromagnetic sublattice ordering. Plotting the total energy as a function of $\theta_{1}$, we again find a roughly sinusoidal dependence, with the minimum at 0 (equivalently $\pi$ ), confirming that the lattices prefer alignment over chirality. ${ }^{14}$

This mean-field spin-1/2 result, along with the preceding approximations finding no order for small $J$, suggests that the low peaks of the cluster diagonalizations (figure 3) represent no chiral correlations beyond the random "background" (footnote 7).

\footnotetext{
14 Insofar as low-order perturbation theory on an infinite lattice may be viewed as a calculation on a small cluster (Gelfand et al., 1990), this mean-field computation, examining antiferromagnetic fluctuations, complements the previous perturbative expansion in ferromagnetic fluctuations.
} 


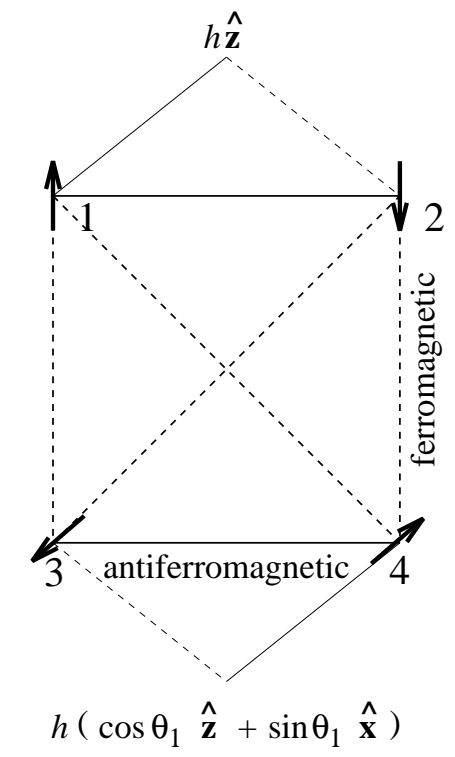

Figure 7. Quantum mean-field theory: consider spins 1 and 2 on one sublattice and 3 and 4 on another. The two spins on each sublattice are linked by an antiferromagnetic bond (solid lines), while spins on opposite lattices are linked ferromagnetically (weakly). To account for antiferromagnetic bonds to spins external to this diagram, we apply mean fields proportional to $h \hat{\mathbf{z}}$ and $h\left(\cos \theta_{1} \hat{\mathbf{z}}+\sin \theta_{1} \hat{\mathbf{x}}\right)$ on each sublattice, determining the self-consistent value of $h$ iteratively.

$$
\text { spin > } 1 \text { order }
$$

We easily repeat the mean-field calculation for all spins 1 through $5 / 2$ on the cluster of figure 7 with the full Hamiltonian, equation (2), and find, in contrast to the spin- $1 / 2$ case, that pairs of sublattices prefer to be mutually perpendicular. (The minimum energy occurs at a relative orientation of $\pm \pi / 2$.) This corroborates the conclusions based on the larger diagonalizations and on spin-wave theory, viz. that spins 1 and higher likely support long-ranged non-trivial chiral order in our model, while spin- $1 / 2$ does not.

If so simple a model as the present, concocted to make chiral ordering easy, fails to show that order for spins- $1 / 2$, we would view cautiously extrapolations of chirality to spin- $1 / 2$ electrons in other square-lattice models. On the other hand, spin-chiral order would not surprise us when electrons combine into spin-1 or higher objects.

\section{Acknowledgements}

This work was supported in part by a Department of Energy Distinguished Postdoctoral Fellowship. 


\section{Appendix: linear spin-wave theory}

\section{choice of expansion parameters}

Classically there are infinitely many ground states, each with a unit cell of eight sites, all related by simple spin rotations or reflections. Choosing one of these, we construct a local right-handed coordinate system on each site in which $S_{i}^{z}$ ( $i$ marking both the site, 0 through 7 , within a unit cell and that cell's coordinate) gives the component of spin in the classically-favored direction, with $q_{i} \equiv S_{i}^{x} / s^{1 / 2}$ and $p_{i} \equiv S_{i}^{y} / s^{1 / 2}$ presumably relatively small deviations ( $s$ is the spin). We choose the names $q$ and $p$ because in fact these deviations are approximately conjugate:

$$
[q, p]=i S_{i}^{z} / s=i \text { minus first-order small corrections. }
$$

From this approximate correspondence follow the semiclassical (linear spin-wave) equations of motion,

$$
\begin{aligned}
& \dot{q}_{i} \approx \frac{\partial H}{\partial p_{i}} \\
& \dot{p}_{i} \approx-\frac{\partial H}{\partial q_{i}} .
\end{aligned}
$$

(Alternatively, consider each spin $\mathbf{S}$ as a symmetric classical top, fixed at the base and with center of mass at $\mathbf{R}$, proportional to $\mathbf{S}$. If the mass is vanishingly small [and the angular velocity infinite, so as to yield angular momentum $\mathbf{S}$ ], we can ignore nutation. Taking the torques resulting from all the other spins in the system to act at $\mathbf{R}$, we have $\dot{\mathbf{S}}=-\mathbf{R} \times \partial H / \partial \mathbf{R}=-\mathbf{S} \times \partial H / \partial \mathbf{S}$, from which we get (9) with exact equality, within this classical approximation.)

Armed with first-order expressions for the $x$ and $y$ components of spin, we can define the linear approximation to the $z$ component as having the form

$$
S_{i}^{z}=s^{\prime}-\frac{1}{2 s^{\prime \prime}}\left(S_{i}^{x 2}+S_{i}^{y 2}\right)
$$

While it would be tempting to fix the constants $s$ and $s^{\prime}$ by the requirement

$$
S^{2}=S^{x^{2}}+S^{y^{2}}+S^{z 2}=s(s+1),
$$

the resulting substitution disagrees with conventional spin-wave theory at small spin (they agree of course in the limit of large $s$ ). The discrepancy apparently arises in the choice of 
which constraints to impose exactly and which only to leading order in the perturbations. Holstein-Primakov satisfies all the important constraints simultaneously; we therefore relate our $q$ and $p$ to the operators of conventional spin-wave theory.

The usual Holstein-Primakov spin-wave treatment identifies the spin operators $S^{-}$and $S^{+}$approximately with Bose creation and annihilation operators $a^{\dagger}$ and $a$, making the deviation from full magnetization $\left(S^{z}=s\right)$ look like a Bose excitation, called a magnon. So long as the magnon-number operator $a^{\dagger} a$ is small compared to the $S^{z}$ quantum number, the approximation is good. Of course, the Bose excitation spectrum is unbounded above, while enough applications of $S^{-}$to any state will annihilate it. There are therefore corrections to the linear theory of quadratic order in the magnon number. Specifically (see Manousakis, 1991),

$$
S_{i}^{+}=\sqrt{2 s}\left(1-a_{i}^{\dagger} a_{i}\right)^{1 / 2} a_{i}=\sqrt{2 s}\left(1-\frac{1}{2} a_{i}^{\dagger} a_{i}+\ldots\right) a_{i} .
$$

Inserting the resulting expressions for $S^{x}$ and $S^{y}$ into the form (10) (discarding pieces of order $\left.\left(a^{\dagger} a\right)^{2} / s\right)$ results in the identification

$$
s^{\prime}=s^{\prime \prime}=s+1 / 2
$$

this choice guarantees that our linear spin-wave theory reproduces the usual results for the antiferromagnet. (We note that Mattis, 1988, equation 5.9, assigns $s^{\prime}=s+1 / 2$ but $s^{\prime \prime}=s$. In contrast, equation (11) implies the assignment $s^{\prime}=s^{\prime \prime}=\sqrt{s(s+1)}$, which however poorly approximates the known magnetization in the $s \rightarrow 1 / 2$ extrapolation.)

We use $q$ and $p$ instead of $a^{\dagger}$ and $a$ because of the relative simplicity of expansions of the quartic terms in the Hamiltonian (2).

\section{solving the classical problem}

We truncate the Hamiltonian at terms quadratic in the operators $p_{i}$ and $q_{i}$; equation (9) then describes a classical, harmonic problem. Consider all the $p$ 's and $q$ 's in a single unit cell (say r), putting them together in the vector

$$
\boldsymbol{\sigma}_{\mathbf{r}}^{\mathrm{T}}=\left(\begin{array}{lllllll}
q_{\mathbf{r}, 0} & q_{\mathbf{r}, 1} & \cdots & q_{\mathbf{r}, 7} & p_{\mathbf{r}, 0} & \cdots & p_{\mathbf{r}, 7}
\end{array}\right)
$$

where $\mathrm{T}$ indicates transposition, and the notation in the subscripts now separates the cell position and the index (0-7) within the unit cell. We assume a spin-wave mode $\mathbf{k}$ with

$$
\sigma_{\mathbf{r}, i}=\operatorname{Real}\left(\sigma_{i}(\mathbf{k}) \exp (i \mathbf{k} \cdot \mathbf{r}-i \omega(\mathbf{k}) t)\right)
$$


( $t$ is time), giving the eigenvalue equation

$$
\omega(\mathbf{k}) \boldsymbol{\sigma}(\mathbf{k})=\mathrm{T}(\mathbf{k}) \boldsymbol{\sigma}(\mathbf{k})
$$

where the non-Hermitian matrix T can be determined directly from (9) and (15) but in fact is also related to the quadratic form $\mathrm{M}$ of the Hamiltonian,

$$
H=\text { constant }+\sum_{\mathbf{k}} \boldsymbol{\sigma}^{\dagger}(\mathbf{k}) \mathrm{M}(\mathbf{k}) \boldsymbol{\sigma}(\mathbf{k})
$$

by

$$
\mathrm{T}(\mathbf{k})=2 i \mathrm{AM}(\mathbf{k})
$$

here $\mathrm{A}$ is the $16 \times 16$ matrix (see Goldstein, p.347)

$$
A=\left(\begin{array}{rr}
0 & 1 \\
-1 & 0
\end{array}\right)
$$

Once we know the eigenvectors of $\mathrm{T},{ }^{15}$ we can normalize them so that each contains energy $\omega / 4$ (since every independent mode corresponds to two time-reversed eigenvectors). Then the substitution (15) gives directly any observable expressed in terms of $q$ 's and $p$ 's. For instance, remembering to use (13) and for a particular choice of coordinate system and labeling of points within the unit cell, one finds on the plaquette whose vertices are 0,3 , and 1 ,

$$
\chi_{031}=s^{3}+\frac{3}{2} s^{2}-\frac{1}{2} s^{2}\left\{\left|q_{0}+p_{3}\right|^{2}+\left|p_{0}-p_{1}\right|^{2}+\left|q_{1}+q_{3}\right|^{2}\right\} .
$$

\section{alternative solution employing a canonical transformation}

An equivalent approach more transparently connected to the standard Holstein-Primakov treatment (or to that of White et al., 1965, but applied directly to the variables $p$ and $q$ ) begins with the Fourier transform, (17). Then $\dot{\boldsymbol{\sigma}}=\mathrm{A} \partial H / \partial \boldsymbol{\sigma}^{\dagger}=2 \mathrm{AM} \boldsymbol{\sigma}$, from which ${ }^{16}$ (16) again follows. We form the columns of the matrix $\mathrm{V}$ from the eigenvectors of $\mathrm{T} ; \mathrm{V}^{-1} \mathrm{TV}$ is diagonal, implying by the equations of motion that $\mathrm{V}^{\dagger} \mathrm{MV}$ is also diagonal. A few lines of

15 It speeds the numerical calculation to rewrite (16) as $2 i \mathrm{M}^{1 / 2} \mathrm{AM}^{1 / 2} \mathbf{y}=\omega \mathbf{y}$, where $\boldsymbol{\sigma}=$ $\mathrm{M}^{-1 / 2} \mathbf{y}$ and $\mathrm{M}^{1 / 2}$ is chosen to be a Hermitian square root of the Hermitian matrix $\mathrm{M}$ (which we can do when, as for most of this work, $M$ is positive definite). This new eigenvector equation in $\mathbf{y}$ is then Hermitian.

16 The 2 comes from the interdependence of $\boldsymbol{\sigma}$ and $\boldsymbol{\sigma}^{\dagger}$. 
algebra demonstrate furthermore that $\mathrm{V}^{\dagger} \mathrm{AV}$ is diagonal. ${ }^{17}$ We can without changing any of the foregoing normalize and permute the columns of $\mathrm{V}$ so that

$$
\begin{aligned}
\mathrm{V}^{-1} \mathrm{TV} & =\operatorname{diag}\left(\omega_{1} \omega_{2} \ldots \omega_{8}-\omega_{1} \ldots-\omega_{8}\right) \\
\text { and } \mathrm{V}^{\dagger} \mathrm{AV} & =\operatorname{diag}\left(\begin{array}{lllll}
-i & -i \ldots-i & \ldots & \ldots & i
\end{array}\right)
\end{aligned}
$$

a step that corresponds to finding a set of uncoupled harmonic-oscillator variables with the masses all set to $1 / 2$ and the spring constants to 2 . Now (21) is not the condition required of a canonical transformation, but $\mathrm{V}^{\prime}=\mathrm{VW}$, where

$$
\mathrm{W}=\frac{1}{\sqrt{2}}\left(\begin{array}{rr}
\mathbf{1} & \mathbf{i} \\
\mathbf{1} & -\mathbf{i}
\end{array}\right)
$$

satisfies the symplectic condition,

$$
\mathrm{V}^{\prime \dagger} \mathrm{AV}^{\prime}=\mathrm{A}
$$

while still diagonalizing M. The transformation

$$
\boldsymbol{\sigma}=\mathrm{V}^{\prime} \boldsymbol{\sigma}^{\prime}
$$

is therefore canonical, meaning that it preserves all the commutators of $p$ 's and $q$ 's. The fact that $\mathrm{V}^{\prime}$ also diagonalizes $\mathrm{M}$ lets us write the Hamiltonian $H$ as the sum of uncoupled oscillators, which we know how to solve.

We now rewrite the original $q$ 's and $p$ 's in terms of the Holstein-Primakov operators $a$ and $a^{\dagger}\left(e . g ., q_{i}(\mathbf{k})=(1 / \sqrt{2})\left(a_{i}^{\dagger}(-\mathbf{k})+a_{i}(\mathbf{k})\right)\right.$, and then derive a simple expression in terms of the matrix $\mathrm{V}^{\prime}$ for the ground-state expectation value $(1 / 2)\left\langle\sigma_{i}^{*} \sigma_{j}\right\rangle$, which we use to evaluate expressions such as (20). The results of course are identical to those we derived first.

17 We assume all the normal-mode frequencies are distinct. Let $\mathbf{v}_{i}$ be an eigenvector of T. Then $M \mathbf{v}_{i}=(1 / 2) i \omega_{i} A \mathbf{v}_{i}$, while $\mathbf{v}_{i}^{\dagger} \mathrm{M}=(1 / 2) i \omega_{i} \mathbf{v}_{i}^{\dagger} \mathrm{A}$ (since frequencies are real and $\mathrm{M}$ Hermitian). For two different eigenvectors, $\mathbf{v}_{i}^{\dagger} \mathrm{M} \mathbf{v}_{j}=(1 / 2) i \omega_{i} \mathbf{v}_{i}^{\dagger} \mathrm{A} \mathbf{v}_{j}$ but also equals $(1 / 2) i \omega_{j} \mathbf{v}_{i}^{\dagger} \mathrm{A} \mathbf{v}_{j}$, implying, since $\omega_{i} \neq \omega_{j}$, that $\mathrm{V}$ diagonalizes $\mathrm{A}$ (and $\mathrm{M}$ as well). 


\section{References}

Affleck, I., T. Kennedy, E.H. Lieb, and H. Tasaki, 1987, Phys. Rev. Lett. 59, 799.

Agterberg, D.F. and M. B. Walker, 1994, Phys. Rev. B50, 563.

Anderson, P.W., 1963, in F. Seitz and D. Turnbull, eds., Solid State Physics 14, 99.

Baskaran, G., 1989, Phys. Rev. Lett. 63, 2524.

Chang, K., I. Affleck, G.W. Hayden, and Z.G. Soos, 1989, J. Phys.:Condens. Matter 1, 153.

Domb, C., 1960, Adv. Phys. 9, 149.

Fisher, M.E. and W. Selke, 1981, Phil. Trans. Roy. Soc. (London) 302, 1.

Gelfand, M.P, R.R.P. Singh, D.A. Huse, J. Stat. Phys. 59, 1093.

Goldstein, H., 1981, Classical Mechanics, 2nd. ed., Addison-Wesley, Reading, Mass.

Gooding, R.J., 1990, "Exact mapping of the $J_{1}, J_{2}$ Frustrated Square QAFM into a Chiral Spin Liquid Hamiltonian," unpublished.

Gor'kov, L.P. and A. Sokol, 1992, Phys. Rev. Lett. 69, 2586.

Harris, E.A. and J. Owen, 1963, Phys. Rev. Lett. 11, 9.

Henley, C.L., 1989, Phys. Rev. Lett. 62, 2056.

Korshunov, S.E., 1993, Phys. Rev. B47, 6165.

Manousakis, E., 1991, Rev. Mod. Phys. 63, 1.

Marston, J.B. and I. Affleck, 1989, Phys. Rev. B39, 11538.

Marston, J.B. and C. Zeng, 1991, J. Appl. Phys. 69, 5962.

Masui, S. and D.D. Betts, 1993, Phys. Rev. B48, 6225.

Mattis, D.C., 1988, The Theory of Magnetism I, corrected 2nd printing, Springer, Berlin.

Mitra, S.N. and K.G. Chakraborty, 1994, J. Phys: Condens. Matter 6, 10533.

Ramasesha, S. and Z.G. Soos, 1984, Intl. J. Quant. Chem. 25, 1003-1021.

Takhtajan, L.A., 1982, Phys. Lett. 87A, 479.

Villain, J., 1979, Z. Phys. B33, 31.

Villain, J., R. Bidaux, J.P. Carton, R. Conte, 1980, J. Phys. (Paris)41, 1263.

Wen, X.-G., F. Wilczek, and A. Zee, 1989, Phys. Rev. B39, 11413.

White, R.M., M. Sparks, I. Ortenburger, 1965, Phys. Rev. 139, A450. 